\title{
Achieving Sharp Resonances in Metamaterials via Engaging “Closed-Modes”
}

\author{
V. A. Fedotov ${ }^{1}$, M. Rose ${ }^{2}$, N. Papasimakis ${ }^{1}$, S. L. Prosvirnin ${ }^{3}$ and N. I. Zheludev ${ }^{1}$ \\ ${ }^{1}$ EPSRC NanoPhotonics Centre, Optoelectronics Research Centre, University of Southampton, SO17 1BJ, UK \\ ${ }^{2}$ School of Physics and Astronomy, University of Southampton, SO17 1BJ, UK \\ ${ }^{3}$ Institute of Radio Astronomy, National Academy of Science, Kharkov, 61002, Ukraine
}

\begin{abstract}
We report on the new way of achieving sharp transmission and reflection resonances in sub-wavelength structured artificial materials.
\end{abstract}

Here we report the first experiential results on a new type of planar metallic metamaterials, which shows exceptionally narrow transmission and reflection stop-bands. The development of structure with a large resonance quality factor and narrow resonant spectral band is crucially important for developing photonics meta-materials such as negative index media, polarization and frequency selective surfaces etc. [1-4].

We achieve the high quality resonances achieved by introducing a symmetry breaking in the shape of metal inclusions forming the meta-material. Such symmetry braking allows the coupling of large volume "closed modes" to free-space. The new type of metamaterial that we report here is formed by a periodic array of identical subwavelength asymmetrically structured planar metallic "particles" placed on a dielectric substrate (see inset to Fig. 1). It exhibits a very sharp resonant response with the quality factor at least one order of magnitude large than that typically encountered in conventional frequency selective structures/surfaces. We show that the nature of such a strong and narrow spectral response can be associated with closed modes, i.e. large volume electromagnetic modes that are weakly coupled to free-space [5]. Coupling to such modes is usually forbidden but they can be excited if, for example, particles have a certain structural asymmetry. Manifestation of the resonant properties depends on the type of the symmetry breaking. For the case of asymmetrically split particles shown in Fig. 1a (a metallic ring split into 2 unequal arcs) the metamaterial exhibits an ultra-narrow resonant reflection stop-band near $5.5 \mathrm{GHz}$ (see Fig. 1b). At this resonance, as shown in the inset to Fig. 1b, two parts of the particle are excited in anti-phase, which dramatically reduces coupling to free-space. "Closed mode" resonance is accompanied by two "conventional" resonances, where excitation of one part of the particle dominates the other (see Fig. 1b). We also found that if the

(a)

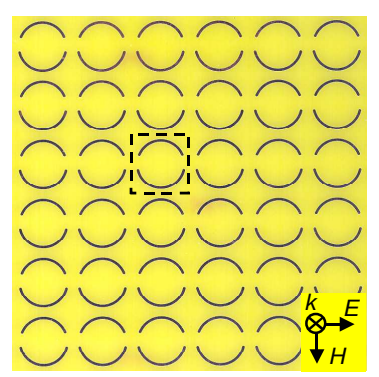

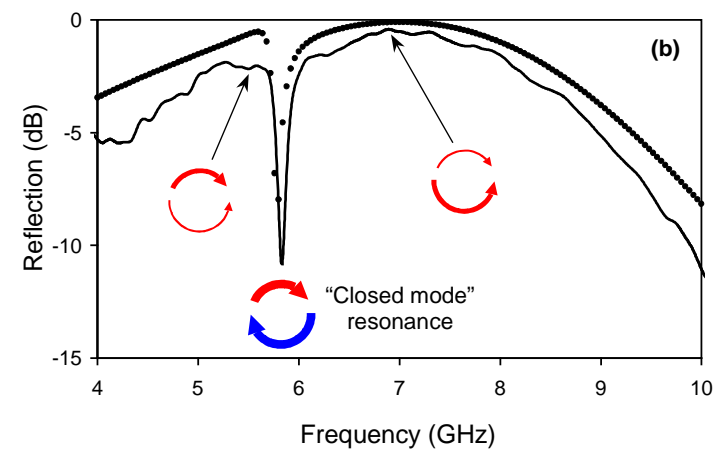

Frequency $(\mathrm{GHz})$

Fig. 1. (a) A fragment of metamaterial with asymmetrically structured particles (two unequal arcs), where dashed line box indicates a $15 \times 15 \mathrm{~mm}$ elementary translational cell of the structure. (b) Intensity of electromagnetic wave reflected from planar metamaterial: solid line - experiment, filled circles theory. Insets present resonant current distributions in the asymmetric particles: arrows show instantaneous directions (phases) of the induced currents while thickness of the lines correspond to relative intensities of the currents. 

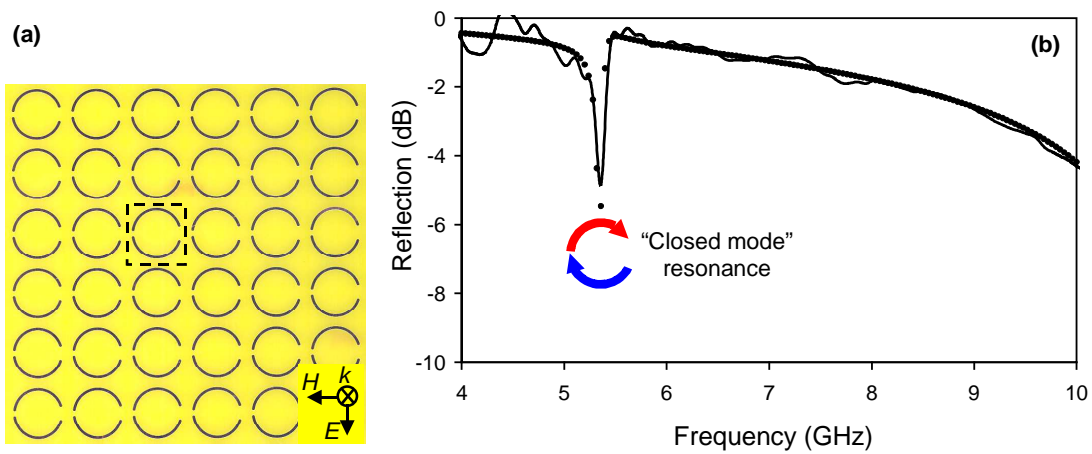

Fig. 2. (a) A fragment of metamaterial with asymmetrically structured particles (two unequal gaps), where dashed line box indicates a $15 \times 15 \mathrm{~mm}$ elementary translational cell of the structure. (b) Intensity of electromagnetic wave transmitted by planar metamaterial: solid line - experiment, filled circles - theory. Inset presents resonant current distributions in the asymmetric particles: arrows show instantaneous directions (phases) of the induced currents while thickness of the lines correspond to relative intensities of the currents.

symmetry breaking is achieved by dividing the ring into two equal arcs with unequal gaps on both sides as presented in Fig. 2a, an ultra-narrow stop-band is seen in transmission near the same frequency (see Fig. 2b). Remarkably, in symmetrical case, i.e. when the arcs as well as splits are equal, no high quality factor resonance can be observed.

In our experiments we used metamaterials that were etched from $35 \mu \mathrm{m}$ copper cladding covering IS620 PCB substrate of $1.6 \mathrm{~mm}$ thickness. Each copper split ring had the radius of $6 \mathrm{~mm}$ and width of $0.8 \mathrm{~mm}$ and occupied a square translation cell of $15 \times 15 \mathrm{~mm}$ (see Fig. 1a and 2a), which ensured that the structures did not diffract electromagnetic radiation for frequencies lower than $20 \mathrm{GHz}$. The overall size of the samples used were approximately $220 \times 220 \mathrm{~mm}$. All the transmission and reflection measurements were performed in an anechoic chamber with the use of broadband horn antennas (Schwarzbeck M.E., model BBHA 9120D) and a vector network analyzer (Agilent, model E8364B). The far-field responses of the asymmetric structures were also modeled using the method of moments [2]. This is a well-established numerical method, which involves solving the integral equation for the surface current in the metal pattern by the field of the incident wave. This is followed by calculations of scattered fields as a superposition of partial spatial waves. The metal pattern is treated as a perfect conductor, while the substrate is assumed to be a lossy dielectric. As it becomes evident from Fig. $1 \mathrm{~b}$ and $2 \mathrm{~b}$ the agreement between the theory and experiment is remarkable.

In summary, we report the first experiential results on a new type of planar metamaterials exhibiting unusually strong transmission and reflection resonances in the microwave part of the spectrum. Spectral response with similar properties is expected in appropriately scaled planar strictures in the IR part of the spectrum, while the ongoing development of nano-fabrication techniques may well lead eventually to the use of the novel planar metamaterials in the visible spectrum. Apart from spectral filtering the sharp nature of "closed mode" resonances can also be used for more accurate "sensing" of the dielectric properties of various substances that are brought in contact with the metamaterial surface, which can have potential applications in moister detectors and gas sensors.

[1] J. C. Vardaxoglou, Frequency Selective Surfaces (Research Studies Press LTD., England, 1997).

[2] S. Prosvirnin, S. Tretyakov, and P. Mladyonov, "Electromagnetic wave diffraction by planar periodic gratings of wavymetal strips", J. Electromagn. Waves Appl. 16, 421 (2002).

[3] D.R.Smith, J.B.Pendry and M.C.K.Wiltshire, "Metamaterials and Negative Refractive Index", Science, 305, 788 (2004)

[4] V. A. Fedotov, P. L. Mladyonov, S. L. Prosvirnin, and N. I. Zheludev, "Planar electromagnetic metamaterial with a fish scale structure", Phys. Rev. E 72, 056613 (2005)

[5] S. Zouhdi, S. Prosvirnin, Advances in Electromagnetics of Complex Media and Metamaterials (Kluwer Academic Publishers, Netherlands, 2003), pp. 281-290 